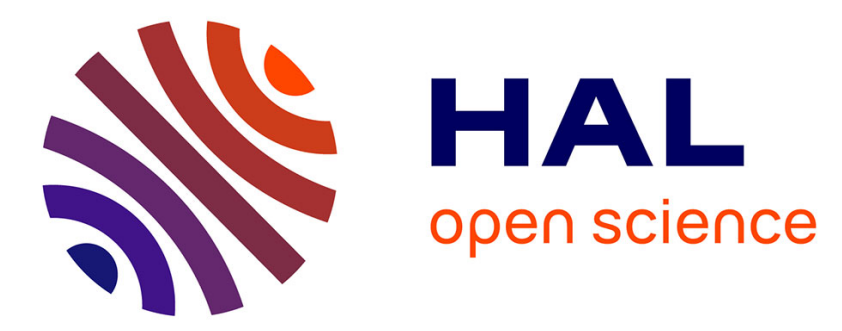

\title{
L'Inde de Ctésias, des sources aux représentations
}

Dominique Lenfant

\section{To cite this version:}

Dominique Lenfant. L'Inde de Ctésias, des sources aux représentations. Topoi Orient - Occident, 1995, 5/2, pp.309-336. halshs-00003909

\section{HAL Id: halshs-00003909 \\ https://shs.hal.science/halshs-00003909}

Submitted on 22 Apr 2005

HAL is a multi-disciplinary open access archive for the deposit and dissemination of scientific research documents, whether they are published or not. The documents may come from teaching and research institutions in France or abroad, or from public or private research centers.
L'archive ouverte pluridisciplinaire HAL, est destinée au dépôt et à la diffusion de documents scientifiques de niveau recherche, publiés ou non, émanant des établissements d'enseignement et de recherche français ou étrangers, des laboratoires publics ou privés. 
Extrait de Topoi 5, 1995.

\section{L'INDE DE CTÉSIAS DES SOURCES AUX REPRÉSENTATIONS ${ }^{1}$}

Le premier auteur grec qui ait traité de l'Inde est, à notre connaissance, Scylax de Caryanda : ce navigateur carien passé au service de Darius avait accompagné le Roi à la conquête du Gandhâra, dans le haut bassin de l'Indus, et fut alors chargé de descendre le cours inférieur du fleuve, aux environs de 520 av. J.-C. ${ }^{z}$. A la suite de cette expédition, il composa un récit sur l'Inde dont ne

1. On ne dispose d'aucune synthèse récente sur les Indica de Ctésias. Outre les études anciennes de C. LASSEN, Indische Altertumskunde II, Leipzig $\left(1874^{2}\right)$, pp. 641-665, de W. REESE, Die griechischen Nachrichten über Indien bis zum Feldzug Alexanders des Grossen; eine Sammlung der Berichte und ihre Untersuchung, Leipzig (1914), pp. 71-91 et de F. JACOBY, s.v. « Ktesias », RE XI (1922), col. 2037-2039, on citera la thèse de J. M. BIGWOOD, Ctesias of Cnidus, Harvard (1964), non publiée (un chapitre sur les Indica) et A. ZAMBrinI, "Gli Indika di Megastene ", Annali della Scuola Normale Superiore di Pisa, $3^{\mathrm{e}}$ série, vol. 12, fasc. 1 (1982), pp. 126-134. Si les Indica furent toujours très décriés et volontiers considérés comme une pure affabulation, les publications récentes tendent à expliquer certains de leurs aspects par des équivalents indiens, réels ou mythologiques. Cf. K. KARTTUNEN, "The Reliability of the Indiaka of Ktesias ", Studia Orientalia, 50, Helsinki (1980), pp. 105-107 ; « A miraculous fountain in India ", Arctos, 19, Helsinki (1985), pp. 55-65; India in early Greek literature, Helsinki (1989) ; J. M. BIGWOOD, «Ctesias' parrot ", Classical Quarterly, 43 (1993), pp. 321-327 ; " Aristotle and the Elephant again », American Journal of Philology, 114 (1993), pp. 537-555.

Ma propre analyse s'inscrit dans le prolongement de ma thèse, qui s'est attachée à établir sur de nouvelles bases le corpus et le texte des fragments, à évaluer la fidélité des principaux auteurs sources, à traduire le texte avec précision, à dégager la cohérence du corpus, à en analyser le contenu et l'apport historique et à retracer l'histoire des lectures de l'œuvre de l'Antiquité à nos jours (Ctésias de Cnide : édition, traduction et commentaire historique des témoignages et fragments, Paris-Sorbonne [1994]). Ce travail doit aboutir à une édition de Ctésias dans la C. U. F. (Les Belles Lettres).

2. Cf. Hérodote, IV,44. L'expédition de reconnaissance effectuée par Scylax fut rapidement suivie par la conquête perse de la vallée de l'Indus vers 518. Cette date 
nous restent que de maigres fragments ${ }^{3}$. Ces derniers laissent à penser qu'il y décrivait les alentours de l'Indus ainsi que diverses peuplades à caractère monstrueux. Si l'Inde fut ensuite évoquée par d'autres prosateurs, comme Hécatée, Hérodote ou Hellanicos, ce fut Ctésias de Cnide qui rédigea, un siècle après Scylax, la première monographie de l'Inde qui ait pour nous quelque consistance ${ }^{4}$. A l'inverse de Scylax, Ctésias n'était pas allé en Inde, mais il avait longuement séjourné dans l'Empire perse et avait aussi lu ses prédécesseurs, Hellanicos ou Hérodote. La distance qui le séparait de l'Inde n'était pas seulement géographique, elle tenait aussi à la médiation de regards étrangers. On s'intéressera ici aux sources grecques et orientales des Indica et à leur interprétation par Ctésias, pour voir en quoi ce tableau de l'Inde s'inscrit en même temps dans une définition des Grecs, de l'Orient et de ce qui fait l'homme en général.

\section{DE L'INDE D'HÉRODOTE À CELLE DE CTÉSIAS}

Etant donné la perte des écrits de Scylax, d'Hécatée et d'Hellanicos, la plus ancienne esquisse d'un tableau de l'Inde figure pour nous dans l'Enquête d'Hérodote. Les Indiens y sont d'abord mentionnés comme composant le

approximative se déduit des inscriptions de Darius : alors que l'inscription de Behistun (entre 520 et 518 ) ne cite pas l'Hindu parmi les provinces soumises au Grand Roi, son nom figure ensuite dans une inscription du mur de soutenement sud de Persépolis (DPe), dont la datation oscille entre 518 et 513. Cf. G. WALSER, Die Völkerschaften auf den Reliefs von Persepolis, Berlin, 1966, p. 31 n. 13 et C. HERRENSCHMIDT, " Nugae antico-persianae ", Achaemenid History IV, Leyde (1990), p. 55.

3. Cf. F. JACOBY, Fragmente der griechischen Historiker, IIIC n709.

4. Les principaux fragments des Indica figurent dans les Fragmente der griechischen Historiker de F. JACOBY (IIIC $\mathrm{n}^{\circ}$ 688) sous les numéros F45 à F52. J'ai retenu quelques fragments supplémentaires dans mon propre corpus (passages d'Elien, de Psellos et de Valère Maxime).

En français, on dispose actuellement d'une traduction du sommaire de Photius par $\mathrm{R}$. HENRY (Photius. La Bibliothèque, Paris, Les Belles Lettres [1959], t. I, codex 72, p. 105-147) et des fragments de JACOBY par J. AUBERGER (Ctésias. Histoires de l'Orient, coll. La Roue à Livres, Paris [1991]).

Les fragments les plus étendus et les plus détaillés sont de Photius et d'Elien, deux témoins qui, tout en étant indépendants, conservent des précisions identiques et semblent être restés très proches de leur modèle, même s'ils tendent à retenir avant tout les données les plus étranges. Cf. J. M. BIGWOoD, "Ctesias' Indica and Photius », Phoenix, 43 (1989), pp. 302-316. 
vingtième des nomes, ou districts de l'Empire perse ${ }^{5}$. Hérodote précise, pour chaque satrapie, le tribut qu'elle remet au Roi de Perse et l'Inde se distingue par la nature et l'importance considérable de celui qu'elle est tenue de verser, trois cent soixante talents de poudre d'or. C'est pourquoi l'évocation de l'Inde intervient à la suite de la liste des tributs, sous couleur d'expliquer l'origine de l'or remis par les Indiens du vingtième nome ${ }^{6}$. Le développement est rapide et partiellement consacré à relater la quête de l'or ${ }^{7}$, mais il confêre à l'Inde une originalité qui ne pouvait que frapper.

Or Ctésias avait lu Hérodote : à plusieurs reprises, dans les Persica, il polémique ouvertement contre lui et le traite de menteur ${ }^{8}$. Un savant de l'époque impériale n'en jugea pas moins utile de dénoncer les plagiats qu'il relevait chez Ctésias ${ }^{9}$ et parmi les supposés plagiés devait bien figurer Hérodote ${ }^{10}$. Enfin, les lecteurs communs aux deux historiens les ont classés dans la même catégorie des fabulateurs ${ }^{11}$. Il paraît donc opportun de s'interroger sur les rapports entre leurs deux récits.

On notera d'abord que bien des évocations de Ctésias paraissent conformes à celles d'Hérodote. L'un et l'autre situent l'Inde à l'extrémité de la terre habitée,

\section{III, 94 .}

6. III, 98-106. L'origine de l'or indien est un sujet fort débattu. Cf. A. FOUCHER, La vieille route de l'Inde de Bactres à Taxila, Paris (1947), p. 216 n. 9, w.W. TARN, The Greeks in Bactria and India, Cambridge (1951), pp. 104-108, G. FUSSMAN, " La notion d'unité indienne dans l'antiquité. I. Les auteurs grecs et latins ", Annuaire du Collège de France. 1984-1985, Paris, pp. 642-643, et, plus récemment, le débat entre P. BERNARD, "Les Indiens de la liste des tributs d'Hérodote ", Studia iranica, 16/2 (1987), pp. 177-190 et W. VogelSANG, «Gold from Dardistan ... ", in P. BRIANT et CL. HERRENSCHMIDT (éd.), Le tribut dans l'empire achéménide... Paris (1989), pp. 157-171 et «Indian antics. A reply to P. Bernard (1987) », Studia iranica, 17/2 (1988), pp. 253-258.

7. III, 102-105.

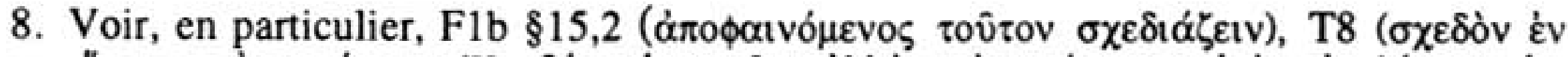

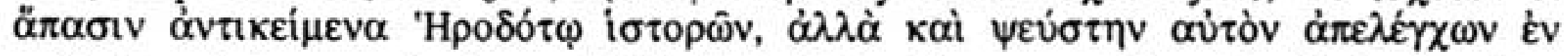

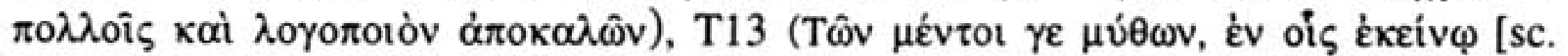

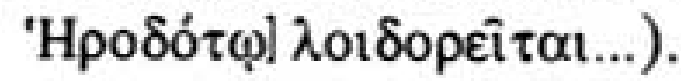

9. C'est Porphyre, cité par Eusèbe (Préparation évangélique, 10,3,23 = T17), qui nous apprend l'existence d'une «lettre de Pollion à Sotéridas Sur les plagiats de Ctésias

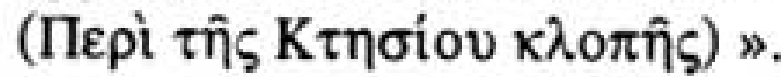

10. On peut aussi songer à Scylax, Hécatée ou Hellanicos. La seule certitude porte sur l'identité du plagiaire et l'on ignore celle des auteurs plagiés ainsi que la matière concernée.

11. Par exemple, Lucien, Histoire véritable, II,31 (non cité par Jacoby) et Strabon, XI,6,3 (T11a). 
qui est bordée à l'est d'une frange désertique ${ }^{12}$. Ils s'accordent à donner les Indiens pour le peuple le plus nombreux du monde ${ }^{13}$. Dans leurs deux récits, l'Inde est peuplée d'ethnies diverses qui pratiquent des mœurs variées : après avoir signalé l'existence de nombreuses peuplades parmi les Indiens ${ }^{14}$, Hérodote distingue nomades et sédentaires, végétariens, mangeurs de poissons crus et consommateurs de viande crue. Et, de son côté, Ctésias évoque différentes ethnies aux mœurs spécifiques: les Pygmées sont des éleveurs et d'excellents archers qui servent dans l'armée du roi ${ }^{15}$, tandis que les Têtes de chiens élèvent des bêtes dont ils consomment le lait, mais vivent aussi de chasse et de cueillette et pratiquent le troc avec le roi des Indiens ${ }^{16}$. Les deux auteurs s'accordent encore sur la taille gigantesque des animaux indiens: Hérodote déclare en termes généraux que "les animaux, quadrupèdes ou volatiles, sont [en Inde] beaucoup plus grands que dans les autres contrées " ${ }^{17}$; Ctésias en propose, quant à lui, plusieurs exemples : les coqs de très grande taille ${ }^{18}$, les chiens si grands qu'ils ne craignent pas d'affronter un lion ${ }^{19}$, les moutons et chèvres de grande taille ${ }^{20}$, le « ver » $(\sigma \kappa \omega \dot{\lambda} \lambda \eta \xi)$ de l'Indus, qui fait sept coudées de long, soit environ trois mètres ${ }^{21}$. Les deux récits mentionnent également le "roseau indien » dont les entre-nœuds fournissent des embarcations ${ }^{22}$ et signalent l'extrême abondance de l'or : d'après Hérodote ${ }^{23}$, le vingtième nome achéménide verse une énorme quantité d'or en tribut au roi de Perse et l'Inde fait partie de ces confins du monde habité censés regorger de ce précieux métal ${ }^{24}$; Ctésias

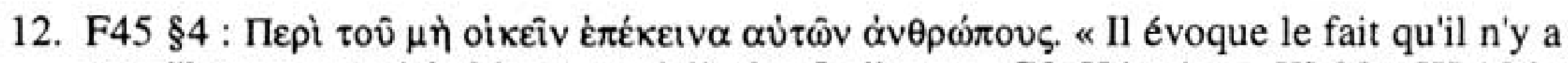
pas d'hommes qui habitent au-delà des Indiens ». Cf. Hérodote, III,98; III,106 ; IV, 40 .

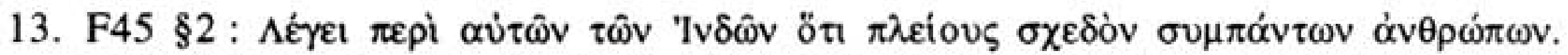
« Des Indiens eux-mêmes, il dit qu'ils sont, ou peu s'en faut, plus nombreux que l'ensemble des hommes ». Cf. Hérodote III, 94 et V,3.

14. Hérodote, III, 98.

15. F45 §22-23.

16. F45 §40-41.

17. Hérodote, III,106.

18. F45 §8.

19. F45 §10.

20. F45 §27 ; Élien, Nature des Animaux, IV,32 (non cité par Jacoby) ; F45i $\alpha$; F45i $\beta$.

21. F45 $\$ 46 ; F 45 r$.

22. F45 $\$ 14$; F45c. Cf. Hérodote, III,98.

23. III,94.

24. III,106, pour l'Inde. Cf. III,114, pour l'Éthiopie et III,115-116, pour le Nord de l'Europe. 
attribue également à l'Inde une grande richesse en or ${ }^{25}$. Enfin, les deux historiens insistent sur les souffrances et les dangers qui entourent la quête de l'or, jalousement gardé dans une région déserte par une faune meurtrière. Les griffons du récit de Ctésias ${ }^{26}$ sont, comme les fourmis géantes de celui d'Hérodote ${ }^{27}$, des bêtes qui extraient l'or du sol et qui menacent de leurs attaques mortelles les chercheurs d'or indiens, contraignant ces derniers à agir dans des conditions très pénibles : la crainte des griffons incite à guetter la nuit noire, comme celle des fourmis la pire des chaleurs.

De nombreux points communs rendent donc ces univers comparables. $\mathrm{Si}$ l'on peut songer à des emprunts de Ctésias à Hérodote, les deux auteurs ont aussi pu recueillir des données similaires et parfois authentiques. Ainsi, le cours moyen de l'Indus est réellement bordé par un désert, le Thar, qui pouvait laisser croire que nul homme n'habitait au-delà.

Si la comparaison s'étend, au-delà du tableau de l'Inde, à l'ensemble de l'histoire d'Hérodote, on constate que des motifs propres à l'ethnographie grecque, situés par Hérodote en d'autres contrées, se retrouvent dans l'univers indien de Ctésias. Hérodote mentionnait ainsi les moutons à longue ou large queue d'Arabie ${ }^{28}$, la longévité exceptionnelle des Ethiopiens ${ }^{29}$, l'or arraché aux griffons par les Arimaspes, à l'extrême Nord de l'Europe ${ }^{30}$, l'absence de porcs sauvages en Libye ${ }^{31}$, les ânes cornus, les créatures à tête de chien et les êtres aux yeux placés sur la poitrine censés peupler les confins occidentaux de cette même Libye ${ }^{32}$. Plutôt que d'imputer à Ctésias le transfert en Inde de traditions

\section{F45 §26.}

26. F45 §26; F45h.

27. III,102-105.

28. Hérodote III,113. Dans les Indica : F45 §27 ; Élien, Nature des animaux, IV,32 (non cité par Jacoby) ; F45i $\alpha ; \mathrm{F} 45 i \beta$. Précisons toutefois que les moutons à large queue ne sont pas des animaux légendaires. Cf. P. BRIANT, État et pasteurs au Moyen-Orient ancien, Paris (1982), p. 137, n. 17.

29. III,114. Dans les Indica: F45 §32; Valère Maxime, Faits et paroles mémorables, VIII,13, ext. 5 (« Aethiopes, quos Herodotus scribit centesimum et vicesimum annum transgredi, et Indi, de quibus Ctesias idem tradit ». Fragment non cité par Jacoby); F45 $\$ 43 ;$ F52.

30. III,116. Dans les Indica: $\mathrm{F} 45$ §26 et F45h.

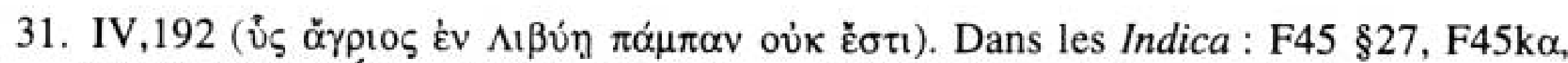
F 45k $\beta$, F45k $\gamma$, Élien, Nature des animaux, XVI,37 (non cité par Jacoby).

32. IV,191. Dans les Indica: F45 §45 et F45q (ânes unicornes) ; F45 §37, F45p $\alpha$, F45p $\beta$,

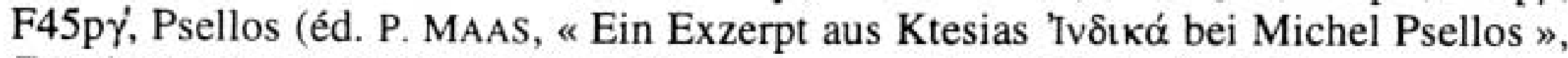
Zqitchrift für vergleichende Sprachforschung aus dem Gebiete der indogermanischen Sprache, Göttingen, 52 [1924], pp. 303-306) (Têtes de chiens), F51a (hommes qui ont les yeux sur la poitrine). 
originellement situées ailleurs, on notera que ces motifs se rattachent quasiment tous aux extrémités du monde, chez Hérodote comme chez Ctésias, et que ces confins du monde suscitaient un type de représentation spécifique ${ }^{33}$.

On a pourtant l'impression que l'Inde de Ctésias tend parfois à rivaliser avec les aspects les plus séduisants de l'Enquête, en particulier avec l'Égypte et les Éthiopiens Longue Vie. Ainsi, l'Indus exerce en Inde le même rôle central que le Nil en Égypte. C'est lui, et non la pluie, qui " arrose " l'Inde ${ }^{34}$. De même, les Éthiopiens Longue Vie semblent à certains égards se réincarner dans les Indiens de Ctésias : mêmes qualités physiques (santé, longévité), mêmes vertus morales (extrême justice), mêmes rapports privilégiés avec le Soleil — tous traits qui se rattachent, là encore, à la représentation grecque des confins du monde.

Entre l'Inde d'Hérodote et celle de Ctésias, il y a donc continuité et rivalité, mais aussi polémique. Plusieurs négations s'expliquent, chez Ctésias, par le désir de réfuter certaines affirmations d'Hérodote. Alors qu'Hérodote faisait venir l'or indien soit de terrains sablonneux hantés par des "fourmis ", soit du sous-sol et des cours d'eau ${ }^{35}$, Ctésias affirme au contraire qu'on ne trouve pas d'or dans les

33. Si des peuples fabuleux habitaient déjà les confins du monde épique, Scylax et Ctésias les situèrent en Inde, tandis qu'Hécatée et Hérodote les virent plus volontiers en Libye ou en Ethiopie (cf. A. DIHLE, «Arabien und Indien ", Hérodote et les peuples non grecs. Entretiens de la Fondation Hardt sur l'Antiquité classique 35, Vandœuvres-Genève [1990], pp. 55-57). On notera pourtant que Scylax est antérieur à Hécatée et à Hérodote et que la localisation indienne est, par conséquent, antérieure à la localisation africaine. La filiation entre Scylax et Ctésias en ce qui concerne les peuples fabuleux (voir infra n. 99) interdit de considérer que Ctésias se contente de transférer en Inde ce qu'Hérodote situait en Libye ou en Ethiopie. Il semble, au contraire, qu'Hérodote et, avant lui, Hécatée aient fait migrer en Afrique des phénomènes jusqu'alors liés à l'Orient. C'est d'un tel processus que résulte, d'après A. LESKY, la représentation des Ethiopiens africains d'Hérodote, qui jouissent, avec leur Table du Soleil et leur source miraculeuse, de privilèges jadis attribués aux Ethiopiens du soleil levant ( Aithiopika ", Hermes 87 [1959], pp. 27-38). Signalons, dans le même sens, que, contrairement à une idée reçue, Ctésias n'a pas transféré en Inde des Pygmées antérieurement situés en Afrique : Hérodote ne donnait aucun nom a la peuplade de petits hommes noirs qu'il situait en Libye (II,32) et Ctésias est, dans la littérature conservée, le premier à conférer une patrie aux Pygmées (cf. P. JANNI, Etnografia e mito. La storia dei Pigmei, Rome [1978], pp. 19-34).

En second lieu, rappelons que si, parmi les peuplades évoquées par Scylax, les unes relèvent de la tradition grecque, d'autres sont bel et bien d'origine indienne (cf. W. REESE, op. cit. [n. 1], pp. 39-52 et infra n. 92). Ces dernières ont donc sans doute contribué à attirer en Inde leurs semblables de Grèce.

34. "O c'est le fleuve qui arrose l'Inde ». Parlant du Nil, Hérodote use à peu près des mêmes termes (II,13) : les Égyptiens, dit-il, « apprirent que tout le pays grec était arrosé par

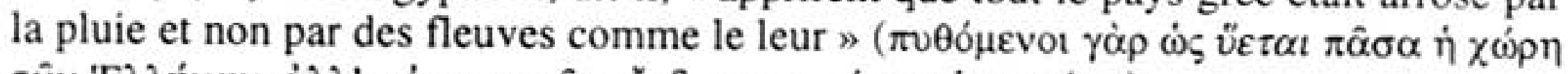

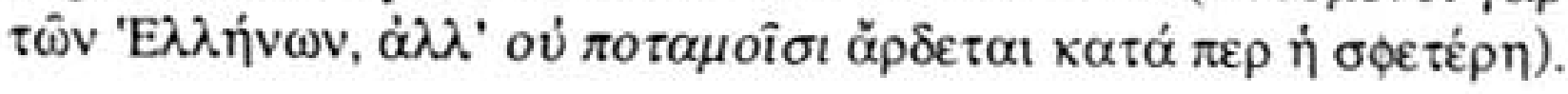


fleuves ${ }^{36}$. D'après Hérodote, les Padéens et les Callaties, peuplades de l'Inde, consommaient la chair de leurs proches défunts ${ }^{37}$; Ctésias prétend à l'inverse que les Indiens ne mangent pas de chair humaine ${ }^{38}$. Et quand il « dit que les Indiens ne sont pas noirs du fait du soleil, mais par nature ${ }^{39}$, il contredit son prédécesseur, qui rendait la chaleur responsable de la couleur de peau noire ${ }^{40}$. En fait, Ctésias se distingue considérablement d'Hérodote et ses écarts ne se fondent pas sur le simple désir de le contredire arbitrairement, mais aussi sur sa connaissance de Scylax et sur des sources orientales.

\section{LA QUESTION DES SOURCES ORIENTALES}

Les ressemblances entre l'Inde de Ctésias et celle d'Hérodote atteignent vite leurs limites. La forme monographique adoptée par Ctésias pose d'emblée l'Inde comme un univers à part, et non comme une simple partie de l'Empire perse, donnant lieu à un développement somme toute marginal. Les peuplades évoquées par les deux historiens ne se ressemblent ni par leur nom ni par leurs caractères physiques ni par leurs mœurs, et il en va de même pour les bêtes.

Ctésias lui-même revendiquait plusieurs sources non littéraires, qui étaient liées à son expérience propre : d'une part ce qu'il avait vu, mais aussi ce qu'il avait goûté ou senti ${ }^{41}$, d'autre part ce qu'il tenait de témoins oculaires ${ }^{42}$. Il va de soi que ces prétentions ne sont pas à prendre pour argent comptant, bien au contraire : c'est au moment où il raconte les choses les plus invraisemblables que l'auteur se réclame de ses sources avec la plus vive insistance. Dans la fameuse

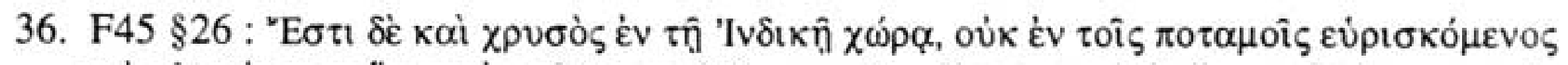

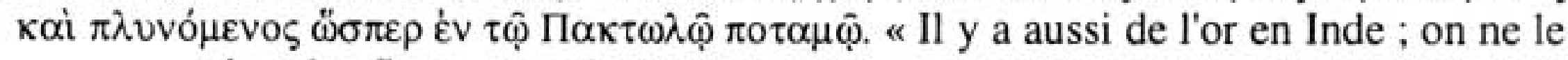
trouve pas dans les fleuves, lavé par les eaux comme dans le Pactole ».

37. Les Padéens : III,99. Les Callaties : III,38.

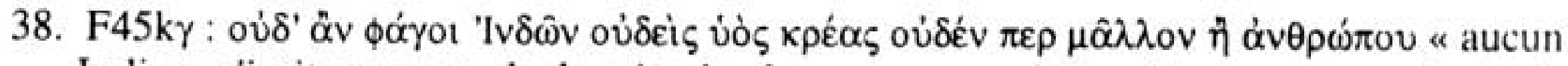
Indien n'irait manger de la viande de porc, pas plus qu'il ne mange de chair humaine ".

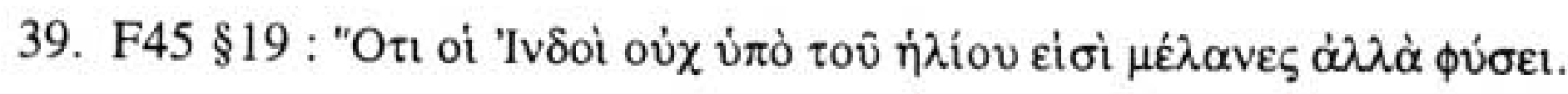

40. II,22. Peu importe qu'il s'agisse ici des riverains des sources du Nil. Peut-être Ctésias croit-il aussi réfuter Hérodote lorsqu'il signale l'existence d'Indiens blancs (F45 \$19

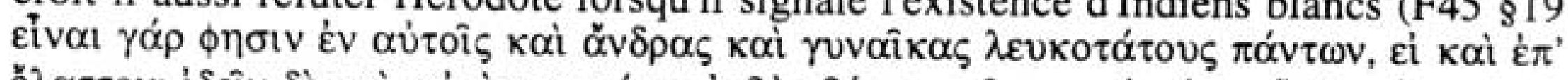

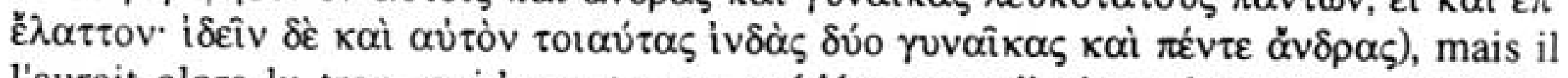
l'aurait alors lu trop rapidement: son prédécesseur disait seulement que tous les Indiens $d u$ Sud avaient la peau noire (III, 101), sans donner de précisions sur les autres.

41. Il dit ainsi avoir senti le parfum tiré du carpion (F45 §47) et avoir goûté le fromage et le vin indiens dont il vante la saveur (F45 $\$ 48)$.

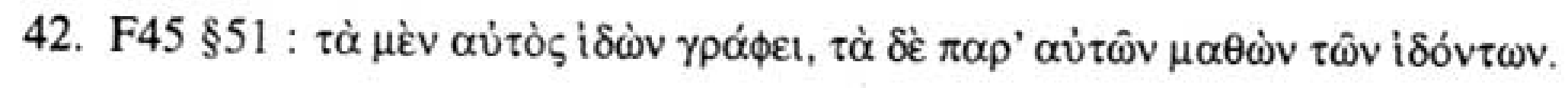


description du martichoras, cette bête au pelage rouge qui a la taille d'un lion, trois rangées de dents, une face humaine, des pattes et des griffes de lion, ainsi qu'une queue hérissée de dards qu'elle lance comme des javelines, queue pourvue, au surplus, d'un dard de scorpion à la piqûre mortelle, Ctésias prétend rapporter les dires des Indiens lorsqu'il dit que les dards de la bête repoussent après usage et il affirme, pour finir, qu'il a vu un martichoras de ses yeux ${ }^{43}$.

De son côté, la documentation orientale ne permet pas de se prononcer de manière décisive sur les sources de Ctésias. Tout d'abord, les confrontations avec les textes indiens sont rarement probantes ou même possibles, ne serait-ce que pour des raisons géographiques et chronologiques. L'Inde de Ctésias est tout au plus la région de l'Indus, au Nord-Ouest du sous-continent indien, dans l'actuel Pakistan, une contrée en marge des principaux foyers de la littérature sanskrite ${ }^{44}$. Qui plus est, la quasi-totalité de cette même.littérature pose d'insolubles problèmes de datation et les estimations oscillent généralement sur plusieurs siècles ${ }^{45}$.

Mais une autre inconnue est à déplorer. Il s'agit du point de vue des Perses sur les Indiens : existait-il des légendes iraniennes sur les Indiens ? C'est ce que donne à penser le récit d'Hérodote qui, lorsqu'il traite des "fourmis » qui menacent les chercheurs d'or indiens, invoque à deux reprises l'autorité des Perses ${ }^{46}$. On est tenté de croire que l'Inde, située à l'extrémité orientale de l'Empire $^{47}$, fut l'ailleurs merveilleux des Perses avant d'être celui des Grecs, mais il est vrai que l'absence de textes littéraires interdit toute certitude en la matière.

Pour juger de la vision perse des Indiens, on ne dispose que de documents peu probants : les listes de peuples de l'Empire mentionnent les satrapies

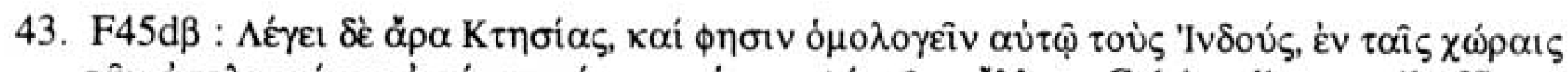

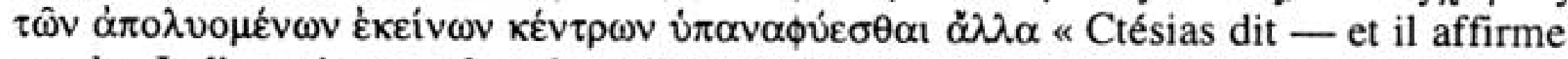
que les Indiens tiennent le même discours que lui - qu'aux endroits où ces dards se

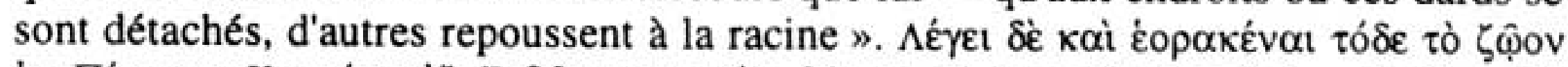

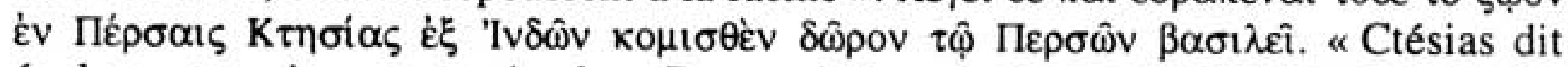
également avoir vu cet animal en Perse, où il avait été apporté par les Indiens en présent au roi de Perse ».

44. Cf. K. KARTTUNEN, India in early Greek literature, Helsinki (1989), p. 6.

45. Ibid., p. 141 sq.

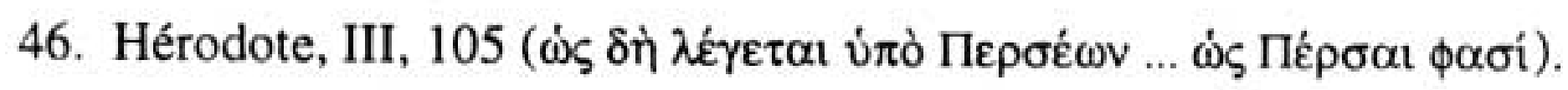

47. Dans les plaques en or trouvées aux coins de l'apadana de Persépolis, Darius exalte l'étendue de son Empire en nommant quatre peuples cités aux quatre points cardinaux. C'est l'Hindu (que l'on situe généralement dans le bassin inférieur de l'Indus, dans la région actuelle du Sind) qui en représente l'extrémité orientale. Cf. G. WALSER, op. cit. (n. 2), p. 33. 
indiennes, et en particulier l'Hindu ${ }^{48}$, et les tablettes des fortifications de Persépolis précisent les rations distribuées à des voyageurs indiens ${ }^{49}$, mais elles ne nous apprennent rien de l'ethnographie perse de l'Inde. Plus éloquents sont les reliefs qui donnent à voir les représentants des divers peuples de l'Empire. Les Indiens figurent parmi les porteurs de trône sculptés sur les tombeaux achéménides (où une légende les identifie sans ambiguïté), parmi les porteurs de trône sculptés dans les palais de Persépolis (Tripylon et salle aux cent colonnes) et parmi les porteurs de présents qui défilent sur le mur de l'escalier est de l'apadana de Persépolis - dans ces deux derniers cas, les archéologues modernes les identifient par comparaison avec les porteurs de trône des tombeaux ${ }^{50}$. Sur les tombeaux achéménides ${ }^{51}$, où les différents peuples se distinguent par leur tenue, leurs armes et leur coiffure, les habitants de la province Hindu (Indiens au sens étroit) présentent le même type vestimentaire que les autres Indiens (au sens large), Sattagydes et Gandariens : les représentants de ces trois provinces indiennes, qui se différencient par quelques détails (barbe, bandeau ...), se démarquent des autres peuples par la nudité de leur torse et par le port d'un pagne ${ }^{52}$. Ces deux caractéristiques se retrouvent à la fois chez les porteurs de trône des palais de Persépolis ${ }^{53}$ et chez les porteurs de présents du défilé de l'apadana ${ }^{54}$. Ces peuples affichent ainsi les conditions

48. L'Hindu est, comme on l'a dit (n. 2), mentionné pour la première fois dans une inscription de Darius à Persépolis (DPe). Il figure ensuite régulièrement dans les diverses listes conservées (inscriptions de Darius au Canal de Suez (DZa-c), à Naqshi Rustam (DNa), à Suse (DSe), inscription de Xerxès dite des daiva (XPh) et inscriptions des divers tombeaux achéménides, qui reproduisent à l'identique la liste du tombeau de Darius). Cf. G. WALSER, op. cit. (n. 2), p. 32 (DZa-c), p. 34 (DNa), p. 37 (DSe), p. 39 (XPh), p. 49 (tombes royales achéménides).

49. R. T. HALlock, Persepolis Fortification Texts, Chicago (1969), pp. 40-45 et W. VogelSANG, " The Achaemenids and India ", Achaemenid History IV. Centre and Periphery, éd. H. SANCISI-WEERdENBURG et A. KUHRT, Leyde (1990), p. 101.

50. On se reportera aux travaux d'E. HERZFELD et surtout d'E. F. SCHMIDT (Persepolis I, The University of Chicago Oriental Institute Publications, vol. 68, Chicago [1953]).

51. Le relief du tombeau de Darius à Naqsh-i Rustam est simplement reproduit sur les cinq autres tombeaux achéménides de Naqsh-i Rustam et de Persépolis.

52. L'épée qu'ils portent en bandoulière dans le dos se retrouve dans d'autres figures, comme celles des Lydiens et des Ioniens ( $\mathrm{n}^{\circ} 22$ et 23 ), de même que la nudité des jambes ou des pieds. On trouvera une description rapide de ces figures dans G. WALSER, op. cit. (n. 2), p. 53, n ${ }^{\circ} 11-13$ et un dessin dépliant en fin de volume (Falttafel 1), partiellement reproduit ici en figure 1.

53. Voir, dans G. WALSER, op. cit. (n. 2), la description des figures 19, 21 et 14 (pp. 6061 et 65 ) et le dessin (pp. 62-63), partiellement reproduit ici (fig. 2).

54. Dans ce dernier cas, il faut admettre de légères restrictions : les membres de la délégation $n^{\circ} 14$, que l'on identifie aux Gandariens, n'ont pas le torse entièrement nu, 
climatiques de leur pays. Ils ne présentent aucune anomalie physique, mais les Indiens « ordinaires " du récit de Ctésias ne sont pas non plus difformes (les Têtes de chiens ou les Pygmées sont présentés comme des peuplades singulières). Rien, dans les reliefs achéménides, ne distingue fondamentalement, les Indiens des autres peuples : ils ne sont qu'une manifestation parmi d'autres de la diversité ethnique de l'Empire. On est également frappé de la relative banalité des présents apportés par les délégations indiennes : un zébu, des javelots, un bouclier, dans le cas des Gandariens ; un âne, deux doubles hâches et deux corbeilles contenant des bourses, dans celui des Indiens. Mis à part l'âne et les corbeilles, de tels présents sont apportés par d'autres délégations et n'ont rien de spécifique ni de très exotique ${ }^{55}$. On peut tout au plus admettre que le contenu énigmatique des bourses laisse place à l'imagination : pierres précieuses, poudre d'or, parfum, poison ... Le récit de Ctésias est en un sens 'conforme à cette représentation des Indiens, lorsqu'il fait allusion aux présents envoyés au roi de Perse par le roi des Indiens ${ }^{56}$, mais les dons que donnent à voir les reliefs sont plus ordinaires qu'un martichoras ${ }^{57}$ et leur banalité contraste avec l'image générale que Ctésias donne de l'Inde. Pour attribuer aux Indica des sources iraniennes, il faut admettre un décalage entre une représentation officielle qui vise à donner la vision d'un Empire riche et harmonieux, où la diversité ethnique n'empêche pas la convergence de tous vers la figure centrale du roi, et, d'autre part, des récits relatifs aux marges de cet Empire.

Or un élément suggère le rôle intermédiaire des Perses dans la perception de l'Inde par Ctésias : il s'agit de noms que l'auteur dit indiens et qui sont de formation iranienne. Le terme de $\mu \alpha \rho \tau \chi o ́ \rho \alpha \varsigma$, que Ctésias traduit par ¿่v $\theta \rho \omega \pi$ ó $\alpha \gamma \circ \varsigma$ " mangeur d'hommes " ${ }^{58}$, peut être rapproché du vieux-perse martiya " homme " et de l'avestique xwar, qui signifie "manger ". De même, quand Ctésias traduit le nom du fleuve $\Sigma \pi \alpha \dot{\beta} \alpha \rho \rho_{\varsigma}$ par $\phi \varepsilon ́ \rho \omega v \pi \alpha \dot{v} \tau \alpha \tau \dot{\alpha} \dot{\alpha} \gamma \alpha \theta \dot{\alpha}$,

mais portent une fine chemise à manches mi-longues (pl. 21, 63, 64, 87 et la description de G. WALSER, op. cit. (n. 2), pp. 89-90) et ceux de la délégation $n^{\circ} 18$, que l'on identifie aux Indiens (au sens restreint), sont conduits par un homme qui porte un surtout fait d'une étoffe légère (pl. 25, 71-73, 86 et la description de G. WALSER pp. 94-95). Voir, ci-contre, figg. 3 à 4.

55. On pourra les comparer à l'ensemble des présents de la procession de l'apadana, récapitulés dans un tableau commode par G. WALSER, op. cit. (n. 2), p. 103 (« Statistik der Geschenke des "Tributzuges" »).

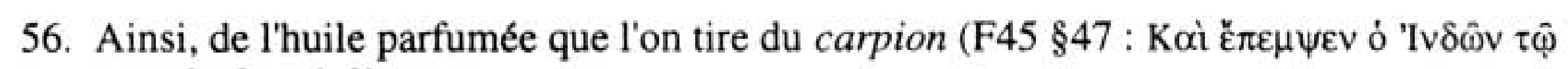

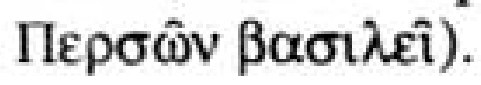

57. Le martichoras est, comme on l'a dit (n. 43), l'un de ces présents envoyés au roi de Perse par le roi des Indiens.

58. F45 $\S 15, F 45 d \beta, F 45 d \gamma$. 
« qui apporte tous les biens " ${ }^{59}$, on peut raisonnablement le faire remonter à une forme iranienne ${ }^{*}$ Vispabara, signifiant « qui apporte tout $" 60$.

Ce qui est sûr, c'est que Ctésias tira une grande part de son inspiration de son séjour en Perse, non seulement de ce qu'il y put entendre, mais de ce qu'il y vit. Ctésias ne prétendait pas être allé en Inde. Chaque fois qu'il précise les circonstances de ses observations, il est question de Babylone, de la Perse ou du grand Roi ${ }^{61}$. Et tout ce qu'il prétend avoir vu est susceptible de voyager : il ne s'agit pas de l'Indus, mais de produits, d'hommes ou de bêtes, comme du vin, des Indiens à la peau blanche ou des éléphants ${ }^{62}$. On l'a vu, sa prétention au témoignage oculaire est loin de constituer une garantie, mais il n'y a pas lieu de douter qu'il ait pu, comme il l'affirme, voir des éléphants à Babylone ou encore contempler des perroquets, des pierres précieuses, des petits singes à longue queue ou des vêtements teints de pourpre - toutes sortes d'échantillons du monde indien donnés pour tels autour de lui.

Mais un autre spectacle put susciter chez lui une veine particulière : il s'agit des représentations de l'art oriental. Tout d'abord, l'iconographie achéménide avait une prédilection marquée pour les êtres hybrides. Avant les Iraniens, les artistes mésopotamiens avaient volontiers associé le buste d'un homme à un corps animal ${ }^{63}$ et les taureaux androcéphales, apparus dans l'art sumérien ${ }^{64}$, vinrent ensuite protéger le palais de Khorsabad ${ }^{65}$. Mais les artistes iraniens développèrent tout particulièrement les représentations d'êtres hybrides : sphinx, taureaux ailés, griffons à tête d'aigle ou à tête de lion ornent couramment sceaux, trônes et chapiteaux, reliefs en pierre et décors en brique émaillée ${ }^{66}$. Sur les

59. F45 §36. Le fleuve appelé " $\Upsilon \pi \alpha \rho \chi \circ \varsigma$ dans le résumé de Photius porte le nom de $\Sigma \pi \alpha ́ \beta \alpha \rho \circ \varsigma$ dans le passage parallèle de Psellos édité par P. MAAS (art. cit. (n. 32)), qu'il convient d'ajouter au corpus des fragments de Ctésias.

60. Cette étymologie iranienne a été proposée par W. SCHULzE dans un addendum à la publication de P. MAAS. La bonne transcription grecque de l'iranien *Vispabara serait

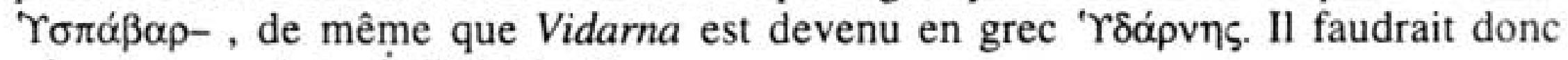
admettre une chute de l'initiale $\Upsilon$-.

61. Il dit, par exemple, avoir vu à Babylone des éléphants et leur cornac indien (F45b), avoir vu un martichoras en Perse ( $\mathrm{F} 45 \mathrm{~d} \beta$ ) et avoir reçu du Roi et de sa mère deux poignards de fer indien ( $\mathrm{F} 45 \S 9$ ).

62. F45 $\$ 48$ (vin), F45 $\$ 19$ (Indiens blancs), F45b (éléphants).

63. Cf. A. PARRot, Sumer, coll. L'Univers des Formes, Paris (1960), pp. 140-141.

64. Ibid., p. 224 pl. 276 et 277.

65. Cf. A. PARrot, Assur, coll. L'Univers des Formes, Paris (1969), pl. 30 pp. 25-26, pl. 34 p. 30 , pl. 35 p. 31.

66. A titre d'exemple, on trouve un certain nombre de sphinx sur les reliefs en pierre de Persépolis (Cf. E. F. SCHMIDT, op. cit. (n. 50), pl. 22, 63, 127), sur les décors en brique de Suse (Sb 3324. Cf. La cité royale de Suse. Découvertes archéologiques en Iran 
briques émaillées de Suse, les sphinx ${ }^{67}$ se présentent comme des lions ailés pourvus d'une tête d'homme barbue, d'oreilles de taureau ornées de boucles d'oreille, avec de hautes coiffures couronnées de plumes et portant trois cornes, tandis que les protomés de lions-griffons ${ }^{68}$ associent la gueule rugissante d'un lion à des cornes et à des oreilles de taureau. Le principe de création de ces êtres hybrides est aussi bien à l'œuvre dans le martichoras ou dans le griffon de Ctésias. Comme les sphinx de Suse, le martichoras rappelle le lion par sa taille, par ses pattes et par ses griffes, il a, tout comme eux, une face humaine et ses oreilles, qui « ressemblent aussi à celles d'un homme, mais sont plus grandes et plus velues ", peuvent se comparer aux oreilles de taureau dont sont pourvus ces sphin ${ }^{69}$. Le dard de scorpion qui couronne sa queue est également une composante des créatures achéménides, tel ce monstre ailé à queue de scorpion qui orne un bas-relief de Persépolis ${ }^{70}$. Quant au griffon des Indica ${ }^{71}$, il rappelle encore plus précisément les diverses composantes des hybrides perses : quadrupède armé de griffes pareilles à celles du lion (comme le sphinx ou le lion-griffon de l'art perse), il est pourvu d'un bec d'aigle (comme les griffons à tête d'aigle) et muni d'ailes et de plumes de diverses couleurs (à l'instar du liongriffon ou du taureau ailé de Suse ${ }^{72}$ ). Décrivant le griffon, Ctésias lui-même présente sa tête comme étant " telle que la peignent et sculptent les artistes " ${ }^{73}$. On peut se demander s'il se réfère à l'art perse, qui lui était sans doute familier, ou à l'art grec, plus connu de ses lecteurs, mais les deux références ne sont pas incompatibles. S'il est vrai que les hybrides sont loin d'être absents de l'art et de

conservées au Musée du Louvre, éd. française révisée par A. Caubet, Paris [1994], pl. 157) et sur les sceaux achéménides - cf. A. U. POPE (éd.), A Survey of Persian Art. From Prehistoric Times to the Present, Oxford (1938), vol. 7, pl. 123 [K]. Des taureaux ailés figurent sur les briques émaillées de Suse et des taureaux androcéphales précèdent la porte des Propylées de Xerxès à Persépolis. Des protomés de griffons à tête d'aigle ornent les chapiteaux de Persépolis (R. GHIRSHMAN, Perse. Proto-Iraniens, Mèdes, Achéménides, coll. L'Univers des Formes, Paris [1963], pl. 267 , p. 218), tandis que des lions-griffons parent les briques de Suse, les extrémités des trônes, les sceaux et les chapiteaux de colonnes (Cf. La cité royale de Suse ... op. cit., pl. 158).

67. Louvre Sb 3324.

68. Louvre Sb 3336.

69. F45d $\beta$.

70. Cf. R. GHIRShMAN, op. cit. (n. 66), pl. 285 p. 238.

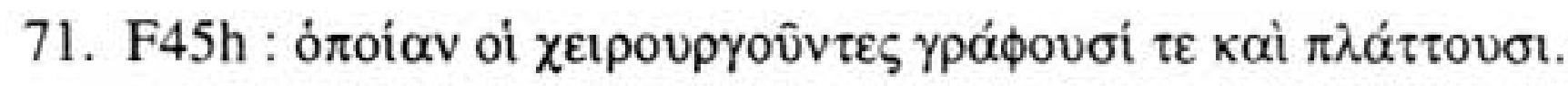

72. Cf. R. GHIRShMAN, op. cit. (n. 66), pl. 191 et 192 p. 142.

73. F45h. 
la mythologie grecs, où se côtoient centaures et sirènes, minotaures et chimères, mais aussi sphinx et griffons, la place de choix que leur accordait l'art perse en ces palais que fréquentait Ctésias a sans nul doute joué un rôle considérable dans l'élaboration de son bestiaire indien.

Ajoutons que l'art aulique achéménide ne distinguait pas « réel » et "fantastique » et n'hésitait pas à représenter le grand Roi en lutte contre le griffon ${ }^{74}$. Quand Ctésias évoque le fer qui, fiché en terre, détourne nuages, grêle et ouragans et dit avoir vu le Roi en faire usage ${ }^{75}$, le rapport entre réel et fantastique n'est pas très différent de ce qu'il est dans la chasse au griffon. Les principes qui sous-tendent l'iconographie achéménide pourraient donc avoir exercé quelque influence sur Ctésias.

Cependant, son récit n'en recèle pas moins des données indiennes que l'on ne trouvait pas dans celui d'Hérodote. Si des données physiques comme la chaleur extrême ou l'existence d'un désert à l'est de l'Indus transparaissaient déjà dans l'Enquête, ce n'est pas le cas de certains usages locaux décrits dans les Indica. Ainsi, la source dont l'eau fait dire la vérité 76 évoque l'ordalie dont la pratique est attestée dans la région à une date très postérieure ${ }^{77}$. De même, les fonctions de justice attribuées au Roi ${ }^{78}$ se retrouvent dans les Lois de Manu, code de lois rédigé aux $\mathrm{II}^{\mathrm{e}}-\mathrm{III}^{\mathrm{e}}$ siècles. Si de telles données sont relativement banales, il en va autrement de l'utilisation des éléphants à la guerre, les uns ayant pour fonction d'effrayer et de charger l'adversaire, les autres de détruire ses remparts ${ }^{79}$ : la première de ces fonctions est attestée à la fois par les récits épiques et par les Lois de Manu ${ }^{80}$, la seconde par l'Arthašhâstra, traité indien de l'art de gouverner ${ }^{81}$.

74. Cf. E. F. SChmidT, Persepolis II, The University of Chicago Oriental Institute Publications, vol. 69, Chicago (1957), pl. 3 sceau n $^{\circ} 1$, décrit p. 18.

75. F45 $§ 9$.

76. F45 $\$ 31$.

77. Le pélerin chinois Faxian rapporte, au Ve siècle ap. J.-C., à propos d'un pays situé à l'Ouest de l'Indus, qu'en cas de doute sur la culpabilité d'un accusé, on lui faisait boire une potion censée lui faire dire la vérité.

78. F45 $\$ 31$.

79. F45 §7, F45b.

80. Cf. C. LASSEN, Indische Altertumskunde, I, Leipzig, $1874^{2}$, p. 355.

81. Áttribué à Kautilya (IVe siècle av. J.-C.), ce texte est daté par la critique moderne du

$1 \mathrm{III}^{\mathrm{e}}$ ou du IV $\mathrm{IV}^{\mathrm{e}}$ siècle de notre ère. Cf. The Kautiliya Arthašhâstra, éd. et trad. R.P. KANGLE, Bombay (1969-72). K. KARTTUNEN signale aussi cet éléphant qui, dans les Jâtaka, brise les portes de Bénarès ( "The reliability of the Indika of Ktesias ", Studia Orientalia, 50, Helsinki [1980], pp. 105-107). 
La faune s'offre aussi à plusieurs recoupements : outre les molosses destinés à défendre les troupeaux ${ }^{82}$ et les troupes de "taureaux sauvages " ${ }^{83}$, qui peuvent évoquer les buffles ravageant les champs indiens ${ }^{84}$, Ctésias donne quelque idée de l'éléphant ${ }^{85}$ et du perroquet ${ }^{86}$. Ce dernier était jusque là inconnu des Grecs, qu'il étonnait encore à l'époque d'Alexandre ${ }^{87}$.

Les notations botaniques sont parfois d'une précision étonnante : le " roseau indien " ${ }^{88}$ rappelle de très près le bambou. Il est "si épais qu'il faut deux hommes les bras tendus pour l'enlacer et aussi haut que le mât d'un navire pouvant charger dix mille amphores ". Il fait " deux brasses d'épaisseur et un seul entre-nœud permet de faire deux cargos ». Or, la tige cylindrique du bambou peut atteindre quelque trente mètres de haut, elle est sectionnée de noeuds cloisonnants et on l'utilise encore de nos jours dans la fabrication de bateaux ${ }^{89}$. Ctésias distingue aussi, "parmi les roseaux, des mâles et des femelles. Le mâle ne contient pas de moelle ${ }^{90}$ et il est très ferme, mais la

\section{F45 §10 et F46a.}

83. F46a.

84. Bubalus bubalis L. Cf. S. H. PRATER, The Book of Indian animals, Bombay $\left(1971^{3}\right)$, p. 210.

85. Hérodote avait seulement mentionné l'éléphant pour l'Éthiopie (III, 97 et 114) et pour les marges de la Libye (IV,191). Les fragments de Ctésias évoquent surtout les emplois de l'éléphant (F45 §7, F45b et, dans les Persica, F1b §16-19 [Sémiramis] et F9 \$7 [Cyrus]), mais il est probable que l'animal faisait l'objet d'une description détaillée que, plus tard, les compilateurs négligèrent de reproduire, tant l'animal était devenu banal. On n'a donc conservé trace que d'un point contesté de ce portrait, qui concerne le sperme des éléphants, censé « durcir, lorsqu'il sèche, au point de devenir pareil à de l'ambre » (F48b). En fait, Aristote, qui fournit de nombreuses précisions sur les éléphants (cf. H. H. SCULLARD, The Elephant in the Greek and Roman World, Cambridge [1974], p. 37), s'est sans doute en partie fondé sur leur description par Ctésias (cf. J. M. BIGWOOD, " Aristotle and the Elephant again ", American Journal of Philology [1993], p. 542 sq.). Mais il ne cite son nom qu'en cas de désaccord.

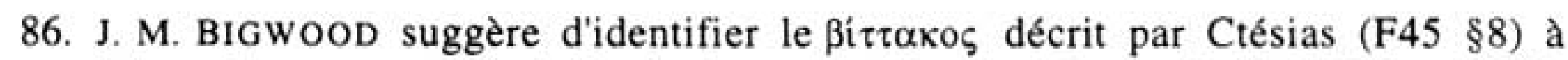
Psittacula cyanocephala (« Ctesias' parrot », Classical Quarterly 43 [1993], pp. 324 325).

87. Arrien (Inde, $\mathrm{XV}, 8)$ se refuse en effet à reproduire la description de l'animal par Néarque, car, si l'oiseau était insolite du temps de ce dernier (fin du IVe siècle av. J.C.), il est parfaitement banal pour les contemporains d'Arrien ( $\mathrm{II}^{\mathrm{e}}$ siècle ap. J.-C.).

88. F45 §14, F45c.

89. Dendrocalamus giganteus peut ainsi atteindre quelque trente-six mètres de haut. Cf. The Wealth of India. A dictionary of Indian Raw Materials and Industrial Products, s.v. Bamboos, vol. 1, Delhi (1948), pp. 145-147.

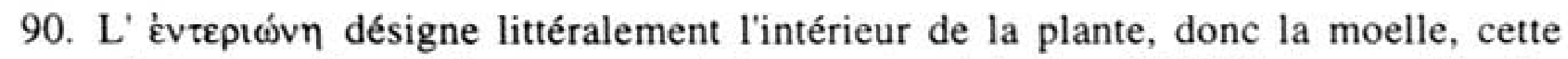
substance molle que l'on trouve parfois au centre de la tige. Cf. Théophraste, Histoire 
femelle en a ". Or, les espèces de bambous se répartissent effectivement en deux types, dont le plus courant présente une tige creuse qui le fait appeler «bambou femelle», tandis que d'autres espèces, à tige pleine, sont appelées " bambous mâles $" 91$.

Mais si les Indica se font l'écho de certaines réalités du monde indien, ils comportent aussi des éléments de sa mythologie. Ainsi, plusieurs des créatures étranges évoquées par Ctésias trouvent leur correspondant dans les textes sanskrits, et en particulier dans l'épopée : les êtres qui n'enfantent qu'une fois (Ekagarbha), ceux qui se couvrent de leurs oreilles (Karnaprâvarana), ceux qui ont un palmier pour jambe (Tâlajangha) ou ceux qui n'ont qu'un pied (Ekapâda ${ }^{92}$. Il est vrai que ce qui n'était, chez les Indiens, que démon ou créature isolée est devenu chez Ctésias ${ }^{93}$ un type ethnique. Mais de telles coïncidences ne peuvent être le fruit du hasard.

Autre analogie frappante : Ctésias évoque une source du nom de Sila dans laquelle rien ne peut flotter et où tout coule à pic ${ }^{94}$; or, on trouve, dans l'épopée indienne, une rivière de même nom, qui constitue la frontière du paradis mythique d'Uttarakuru et dans laquelle tout se change en pierre ${ }^{95}$.

Le " ver de l'Indus " nous fournit encore un exemple de parallèle saisissant avec une créature de la mythologie indienne, le serpent Shesha. Le « ver de l'Indus " est décrit comme un reptile géant que l'on pend, après l'avoir capturé, afin que s'écoule de son corps une huile incendiaire ${ }^{96}$. K. Karttunen a mis en lumière ses ressemblances avec le serpent Shesha qui, à chaque kalpa (période d'une création de l'univers), vomit le feu qui détruit tout et que l'on représente parfois pendu à un arbre, en train de déverser son poison incendiaire ${ }^{97}$.

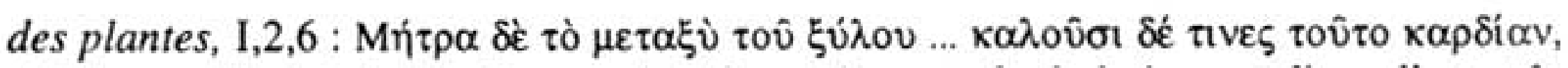
oi $\delta^{\prime} \varepsilon \dot{\varepsilon} v \varepsilon \rho \omega^{\prime} v \eta v$ ( « la moelle constitue la partie centrale du bois ... on l'appelle tantôt le cœur, tantôt la substance interne ").

91. Cf. The Wealth of India, op. cit. (n. 89), p. 145.

92. Cf. C. LASSEN, op. cit. (n. 80), II, p. 654 sq. et J. ANDRÉ-J. FILLIOZAT, L'Inde vue de Rome, n. 121 p. 355 , qui renvoient en particulier au Harivamsha (9553) et au Mahâbhârata, II, 28, 44 et VI, 47, 13.

93. Ou plutôt, avant lui, chez Scylax.

94. F47a, F47b.

95. Cf. C. LASSEN, op. cit. (n. 80), II, p. 657, J. SACHSE, « Le mythe de Silâ, fleuve indien ", EOS, 70 (1982), pp. 237-241 et la mise au point effectuée par K. KARTTUNEN, «A miraculous fountain in India ", Arctos, XIX, Helsinki (1985), pp. 55-65 et India..., op. cit. (n. 44), pp. 186-189.

96. F45 §46 et F45r.

97. K. KARTTUNEN, India..., op. cit. (n. 44), pp. 190-191. 
Malgré les écarts chronologiques, géographiques et sémantiques qui séparent ces différentes sources, les analogies paraissent trop réelles et plurielles pour que l'on admette de simples coïncidences. Du reste, les «fourmis " d'Hérodote sont là pour attester la large diffusion de certaines légendes indiennes ${ }^{98}$. Il est impossible de dire par quel intermédiaire ces données parvinrent à Ctésias : les sources grecques elles-mêmes ont pu jouer leur rôle, puisque Scylax, dont le récit était sans doute disponible à la cour perse, avait déjà évoqué les diverses créatures mythiques à caractère monstrueux ${ }^{99}$ et que Hellanicos, dont Ctésias connaissait l'œuvre ${ }^{100}$, avait déjà décrit la fontaine Sila ${ }^{101}$.

Ces divers échos, même s'ils n'avaient pas été déformés, avaient de quoi étonner un Grec et conféraient à l'univers indien une couleur particulière. Les dimensions de l'Indus, l'étouffante chaleur, la taille d'un animal tel que l'éléphant pouvaient inspirer l'idée qu'en Inde tout est démesuré et confirmer ainsi un topos préexistant de l'image grecque des confins du monde. Un animal doué de parole comme le perroquet pouvait faire figure d'hybride, bête pourvue d'attributs humains, et donnait ainsi quelque crédibilité à d'autres créatures étranges, mais, elles, purement mythiques. C'est ainsi que dut surgir l'idée qu'en Inde tout était excessif et bizarre, que tout y remettait en cause les normes et concepts généralement admis. Ainsi put germer, dans l'esprit de Ctésias, un modèle propre à façonner le reste de l'univers indien. Enveloppé, déformé ou réinterprété, le noyau d'origine indienne suscita des développements fantastiques.

\section{LES PARAMÈTRES DE L'ALTÉRITÉ INDIENNE}

Les échos du monde indien frappèrent Ctésias ou ses sources par des qualités propres qui servirent de paradigmes. Parmi ces caractères figurent la démesure, la couleur, l'hybridisme et le monstrueux.

98. Hérodote évoque des fourmis plus grandes qu'un renard, censées creuser le sol et rapporter à sa surface un sable mêlé d'or (III,102). On a, depuis longtemps, rapproché ce récit d'un passage du Mahâbhârata (II,1860), qui mentionne, parmi les tributs versés au prince Yudhishthira, "l'or des fourmis » (pipîlika), qui tire son nom des fourmis (pipilika) permettant de l'extraire. Pour la bibliographie afférente, on se reportera à l'article d'A. ZAMBRINI, loc. cit. (n. 1), p. 121 n. 34.

99. Tzetzès, Chiliades, VII, 629-649 Kiessling $=$ FGrHist 709 F7b.

100. F16 §62.

101. FGrHist $4 \mathrm{~F} 190$. 


\section{La démesure}

Un animal tel que l'éléphant donnait l'exemple du gigantisme indien. Mais tout, dans l'Inde de Ctésias, paraît démesuré et excessif : les dimensions de l'Indus ; la chaleur extrême ; la très grande taille des animaux ; les dimensions du territoire indien, qui égale à lui seul le reste de l'Asie ; sa population, qui dépasse celle du reste du monde ... Si le gigantisme caractérisait déjà les confins du monde tels que les peignait Hérodote, d'autres changements d'échelle paraissent plus originaux.

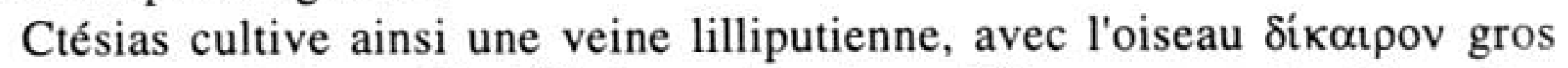
comme un œuf de perdrix ${ }^{102}$, les petits singes ${ }^{103}$ et surtout les Pygmées, hommes qui mesurent entre une coudée et demie et deux coudées, soit quelque soixante-dix centimètres ${ }^{104}$.

Enfin, outre le géant et le minuscule, c'est la disproportion qui marque le monde indien. Le petit singe à longue queue, de type langur, put faire office de paradigme. Mais ce sont les Pygmées qui offrent le plus bel exemple de disprorportion : tout en étant très petits, ils ont de longs cheveux qui leur vont jusqu'aux genoux, un sexe qui leur atteint les chevilles et une longue barbe qui leur touche les pieds ${ }^{105}$.

\section{La couleur}

Dans son ethnographie, Hérodote ignorait pratiquement la couleur. La palette des Indica est au contraire bigarrée, en particulier dans les descriptions d'animaux. Il se peut que des spectacles orientaux aient contribué à inspirer ce bariolage, qu'il s'agisse d'un oiseau tel que le perroquet ou des produits de l'art achéménide : les décors en brique émaillée étaient polychromes, tout comme l'étaient, à l'origine, les reliefs en pierre.

Les couleurs qui teignent l'univers indien de Ctésias sont cependant toujours les mêmes : outre le noir ${ }^{106}$ et le blanc ${ }^{107}$, on relève la couleur de l'ambre ${ }^{108}$

102. F45 §34, F45m.

103. F45 §8.

104. F45 §21; F45f $\alpha$

105. Ibidem.

106. Barbe noire du bittacos (F45 §8), plumes noires du griffon ( $\mathrm{F} 45 \S 26$; F45h), partie noire de la corne de l'âne sauvage (F45 $\S 45 ; \mathrm{F} 45 \mathrm{q})$, poison noir que l'on tire du ,serpent pourpre (F45 $\$ 33 ; \mathrm{F} 451)$.

, 107. Ailes blanches du griffon (F45h), tête blanche du serpent pourpre (F45 $\$ 33 ; \mathrm{F} 451)$, corps blanc et corne partiellement blanche de l'âne sauvage (F45 §45; F45q).

108. Couleur de l'un des poisons tirés du serpent pourpre (F45 $\S 33 ; \mathrm{F} 451)$ et du sperme d'éléphant séché (F48b). 


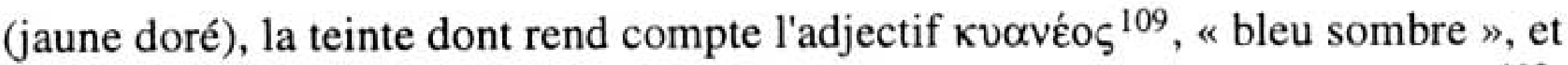
le rouge, couleur de la pourpre, mais aussi de l'arsenic, du cinabre et du sang ${ }^{110}$. Ce sont des couleurs lourdes de connotations. L'ambre évoque la richesse

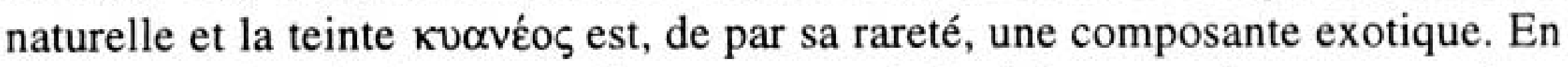
tant que couleur de la pourpre, le rouge connote la richesse, mais comme couleur du cinabre (ou sulfure de mercure), il évoque le danger de mort ${ }^{111}$. Sa suprématie contribue sans nul doute à rendre l'Inde étrange.

\section{Les hybrides}

Les animaux sont souvent décrits par comparaison avec d'autres espèces auxquelles ils paraissent emprunter telle ou telle partie de leur corps. Le griffon est, par exemple, pourvu d'un bec d'aigle, il a les pattes et les griffes d'un lion et la taille d'un loup. Le martichoras est un composé de lion, d'homme, de biche et de scorpion.

Aux origines de telles créatures se trouve d'abord une technique de description qui part d'animaux connus du lecteur pour lui donner à voir une bête inconnue. Hérodote décrivait ainsi l'hippopotame comme un composé de boeuf et de cheval ${ }^{112}$ et Diodore affina par la suite en lui reconnaissant des attributs du sanglier ou de l'éléphant ${ }^{113}$. Une telle description ne procède donc pas nécessairement d'une création fantastique, mais il est improbable que de tels montages aient suscité dans l'esprit du lecteur une image très proche de l'hippopotame. Rappelons qu'une telle veine fut aussi stimulée chez Ctésias par l'art animalier achéménide.

109. Couleur du bittacos ( $\mathrm{F} 45 \S 8$ ), de certaines plumes du griffon ( $\mathrm{F} 45 \mathrm{~h})$ et des yeux de l'âne sauvage (F45 §45).

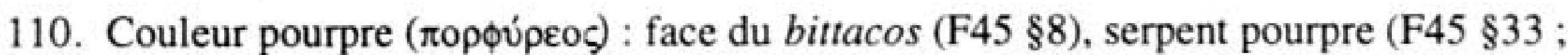
F451), tête de l'âne unicorne (F45 §45; F45q), fleur pourpre (F45 §38); (申oเvıкov̂) : corne de l'âne sauvage (F45 $\S 45 ; \mathrm{F} 45 \mathrm{q})$. Couleur de l'arsenic : dicairon (F45m :

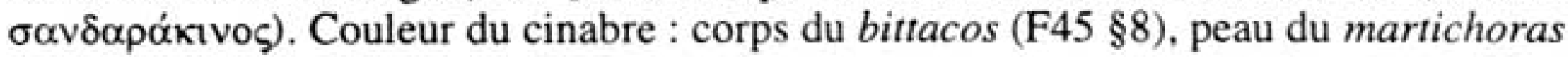
(F45 $\S 15 ; \mathrm{F} 45 \mathrm{~d} \alpha, \beta)$, insectes vivant sur l'arbre producteur d'ambre (F45 §39), astragale de l'âne sauvage (F45 §45). Couleur du sang : peau du martichoras (F45d $\delta$ : colore sanguineo. L'image est de Pline, mais confirme la connotation de danger).

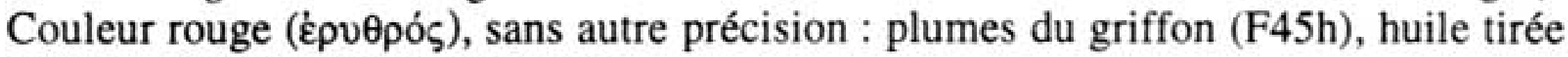
du carpion ( $\mathrm{F} 45 \$ 47$ : vं

111. Sur les connotations du cinabre, voir P. ELLINGER, Recherches sur les "situations extrêmes" dans la mythologie d'Artémis et la pensée religieuse grecque, Thèse d'Etat (1988), pp. $377-378$ et n. 169 p. 469 , n. 175 pp. $470-472$.

112. Hérodote, II,71.

113. Diodore, $\mathrm{I}, 35,8$. 


\section{Les monstres}

J'entends par monstres des créatures humaines présentant des aberrations anatomiques qui ne les rapprochent même plus des bêtes comme dans le cas des hybrides. Des figures de la mythologie indienne purent servir de modèle : on a déjà évoqué les êtres qui n'ont qu'un pied, ceux qui ont un palmier pour jambe, ceux qui se couvrent de leurs oreilles et ceux qui n'enfantent qu'une fois. De telles figures se caractérisent par une anomalie physique, qui représente, par rapport à l'homme normal, un manque, une restriction, un excès ou une inversion. Ce principe inspire la description des peuplades singulières de l'Inde de Ctésias. C'est un manque qui caractérise les êtres privés d'anus ou les hommes sans cou ; c'est une restriction qui marque les Pygmées, les créatures qui n'enfantent qu'une fois ou celles qui n'ont qu'une jambe ; c'est un excès qui frappe les hommes polydactyles, ceux dont les oreilles leur couvrent dos et bras, ceux dont les pieds sont si larges qu'ils leur servent d'ombrelle ou encore les Pygmées dont les poils descendent jusqu'aux pieds ; c'est enfin l'inversion d'un processus biologique qui caractérise les enfants qui naissent pourvus de dents et de cheveux blancs, lesquels noircissent quand ils atteignent l'âge mur.

Certains de ces aspects rejoignent des schémas imaginaires relatifs aux confins du monde : le gigantisme ou le monstrueux se retrouvent chez Hérodote à propos de l'Éthiopie (à l'extrême sud), des confins de la Libye ou à propos de l'extrême Nord.

De plus, comme ces divers caractères ont des relents mythiques, l'Inde tend à devenir un réceptacle de mythes. Ctésias y place des légendes que l'on situait habituellement en d'autres lieux - comme les griffons gardiens de l'or, que l'on imaginait avant dans l'Extrême Nord ${ }^{114}$, ou le fleuve qui charrie de l'ambre : Ctésias situe en Inde l'Eridanos ${ }^{115}$, fleuve mythique de l'Extrême Occident dont Hérodote avait nié l'existence ${ }^{116}$. Par ce surcroît d'investissement mythique, l'évocation de l'Inde devient prétexte à exprimer des conceptions grecques, qui ont d'abord trait à la définition de l'homme.

\section{L'HUMANITÉ AU MIROIR DE L'INDE}

L'ethnographie grecque est tributaire des structures d'un imaginaire collectif, qui en font l'expression d'une interrogation proprement hellénique sur

114, Hérodote, III,116, IV,13 et 27, qui se fonde sur le poème d'Aristéas de Proconnèse (VII ${ }^{\mathrm{e}}$ siècle).

115. F65. Peut-être faut-il voir dans l'Hyparchos, fleuve qui charrie de l'ambre (F45 §36), un avatar de l'Eridanos.

116. Hérodote, III,115. 
la définition de l'homme et l'identité grecque ${ }^{117}$. Or, ce phénomène est particulièrement sensible dans la peinture des confins du monde dont l'Inde constitue un éminent exemple.

\section{L'Inde comme haut lieu du primitivisme}

L'Inde de Ctésias est un univers où la nature est reine. Or, le milieu naturel y est tout à la fois la source des pires dangers et celle des dons les plus précieux. On y trouve conjointement les conditions de l'Age d'or et les dangers de la nature sauvage ${ }^{118}$. De l'Age d'or on retrouve les productions spontanées de la nature (les unes sources de richesse, les autres de nourriture), les ressources contre les maladies, la grande longévité et l'extrême justice des hommes. Quant aux dangers mortels de la nature sauvage, ils sont dus au climat (la chaleur suffocante fait périr quantité d'Indiens) et à la redoutable faune (martichoras, griffon, serpent pourpre, âne sauvage).

Certains endroits sont explicitement marqués de cette ambivalence : le Mont Sardo regorge de pierres précieuses, d'onyx et de sardoine, mais il est aussi hanté par le très vénéneux serpent pourpre et frappé de la pire des chaleurs ${ }^{119}$; de même, les montagnes qui recèlent l'or indien sont aussi le séjour des terribles griffons ${ }^{120}$.

En cumulant des traits de l'Age d'Or et des périls de la nature sauvage, l'Inde réunit en elle les deux représentations antithétiques de l'humanité primitive - contre lesquelles les Grecs définirent l'humanité normale et leur propre identité. L'ethnographie des confins du monde situe ainsi dans un lointain espace ce que l'histoire mythique des origines fait reculer dans le temps et qui n'est rien d'autre que les envers possibles du hic et nunc des Grecs. Ce processus était déjà à l'œuvre dans l'ethnographie de l'Enquête, mais le récit de Ctésias, contrairement à celui d'Hérodote, fait en même temps vaciller la définition de l'humain par la place qu'il accorde aux créatures hybrides et monstrueuses.

117. Outre les travaux de F. HARTOG, Le Miroir d'Hérodote. Essai sur la représentation de l'autre, Paris $\left(1991^{2}\right)$, on se reportera aux études de C. JACOB, qui conclut en ces termes son analyse du Périple d'Hannon: «l'inventaire des formes de vie humaine débouche toujours sur une interrogation des critères de l'humanité et de la civilisation, sur une question d'identité, qui peut être posée implicitement ou explicitement » (Géographie et ethnographie en Grèce ancienne, Paris [1991], p. 84).

118. Je ne développerai pas ici une analyse déjà exposée dans un article intitulé "Milieu naturel et différences ethniques dans la pensée grecque classique", Ktèma 16, Strasbourg (sous presse).

119. F45 § 11 et $33 ; \mathrm{F} 451$.

120. F45 §26 et F45h. 


\section{Monstres et hybrides, entre l'homme et la bête}

Bien des animaux indiens ne se contentent pas de leur apparence hybride. Ils ont aussi cette particularité de posséder des traits humains. Le perroquet a pu inspirer le principe de cette humanisation des bêtes : le bittacos est un oiseau doté de la parole et capable de parler n'importe quelle langue " tout comme un homme ". De même, le martichoras ressemble à l'homme par sa face, ses yeux et ses oreilles, comme par ses manières d'archer sace.

Inversement, certaines peuplades de l'Inde se caractérisent par leur ressemblance partielle avec une espèce animale : les Cynocéphales tiennent du chien par l'aspect de leur tête, leurs aboiements et leur manière de s'accoupler ; mais ils comprennent la langue indienne et s'expriment par des gestes comme des muets. L'hybridisme estompe ainsi les frontières entre l'homme et l'animal. Compilant les Indica, Photius présente les Têtes de chiens comme des hommes, alors qu'Elien les évoque parmi les animaux.

De plus, il est parmi les Indiens des peuplades qui se distinguent par des anomalies physiques spécifiques : Pygmées petits et laids, hommes sans anus, femmes qui n'enfantent qu'une fois, enfants qui naissent avec des dents et des cheveux blancs, êtres ayant des membres de huit doigts, hommes aux oreilles gigantesques ou n'ayant qu'une seule jambe, créatures qui se mettent à l'ombre de leurs larges pieds.

Ctésias se distingue ici d'Hérodote, qui refusait d'ajouter foi à ce type de légendes. Dans l'Enquête, l'homme et l'animal se distinguent clairement. Quand les frontières s'estompent, Hérodote recule, il émet des réserves ou affirme son rejet. Il refuse de croire qu'il y ait des hommes aux pieds de chèvre ou aux yeux placés dans la poitrine ${ }^{121}$. Si Ctésias cultive, quant à lui, les aberrations anatomiques, c'est sans doute imputable à ses sources orientales, à son orientation d'esprit, peu critique et soucieuse d'étonner, mais aussi à son intérêt pour le corps, sans doute lié à sa formation médicale ${ }^{122}$. Hérodote s'intéresse peu au physique des hommes ${ }^{123}$. Quand il dépeint des peuples étranges ou bestiaux, ce sont leurs mœurs qui les rendent tels. Au contraire, Ctésias transfere la question de l'identité humaine sur le plan anatomique.

\section{IV, 25, IV, 191 .}

122. Une comparaison de l'ethnographie d'Hérodote et du traité hippocratique Des Airs, des Eaux, et des Lieux, conduite dans le cadre d'un mémoire de maîtrise, m'a permis 'de constater sur ce point le contraste entre un médecin épris de notations physiques et un Hérodote qui ne s'intéresse le plus souvent au corps que lorsqu'il est le support d'un usage. Pour une comparaison plus générale de ces deux ouvrages, de leur méthode et de leur esprit, cf. J. JouANNA, Hippocrate, Paris (1992), p. 319 sq.

123. Sur les précisions physiques dans l'ethnographie d'Hérodote, cf. D. LENFANT, art. cit. (n. 118), n. 6. 
La peinture de l'Inde progresse vers une étrangeté croissante, selon un processus courant dans la littérature ethnographique ${ }^{124}$ : les Pygmées sont étrangement petits et disposent d'une curieuse pilosité, mais ils ne sont pas comparés à des bêtes et parlent encore la langue des Indiens ; les Cynocéphales sont, eux, comparés à des bêtes et ils ne parlent plus, ils comprennent seulement la langue des Indiens; les peuplades évoquées ensuite présentent des anomalies croissantes, qui les éloignent de l'humanité sans les rapprocher de l'animal, mais elles fournissent toujours des contingents au roi. Comment dire où commence l'humanité et où elle s'arrête ?

Ctésias brouille les pistes. Tout d'abord, le corps des monstres présente des caractères prêtés d'ordinaire aux animaux. Dans le mythe de Prométhée tel que le rapporte Platon dans le Protagoras ${ }^{125}$, Epiméthée pourvoit les animaux de défenses naturelles contre les intempéries. «Il les habillait d'une épaisse fourrure aussi bien que de solides carapaces, propres à les protéger contre le froid, mais capables d'en faire autant contre les brûlantes chaleurs ; sans compter que, quand ils iraient se coucher, cela constituerait aussi une couverture, qui pour chacun serait la sienne et qui ferait naturellement partie de lui-même ». Les peuplades indiennes de Ctésias disposent précisément de ce type d'attributs : les Pygmées ont le corps couvert de longs poils qui leur servent de vêtements, d'autres se protègent de la chaleur avec leurs larges pieds ou leurs grandes oreilles.

Mais Ctésias efface surtout les repères en cantonnant la monstruosité à l'aspect physique : ces peuplades physiquement aberrantes ont cependant en commun une très grande justice. Les Têtes de chiens peuvent à la fois s'accoupler comme des bêtes et constituer des modèles de justice. Or, ce sont deux traits inconciliables dans l'ethnographie d'Hérodote : les peuples que ce dernier situe aux extrémités du monde habité sont soit des sauvages qui s'accouplent comme les bêtes (certains Libyens, certains Indiens ou Caucasiens ${ }^{126}$ ) soit des hommes d'une justice idéale (les Ethiopiens Longue vie). Ctésias confond au contraire ces deux catégories ${ }^{127}$.

Hérodote condamne le comportement bestial chez les hommes et rejette la référence à la nature. La nette distinction qu'il opère implicitement entre l'homme et l'animal préfigure les efforts de conceptualisation de Platon ou d'Aristote s'employant à préciser les prérogatives de l'homme. Au contraire, les

124. Voir, par exemple, le Périple d'Hannon ou l'Inde d'Arrien.

125. Platon, Protagoras, 321 a (trad. L. Robin).

126. IV,180; III,101; I,203.

127. En juxtaposant ces deux traits dans son texte, Ctésias montre qu'il est conscient de

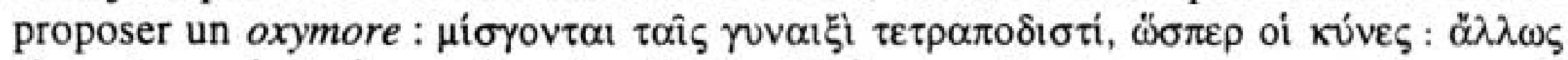

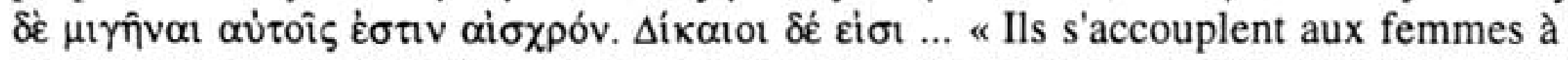
quatre pattes, comme les chiens. S'accoupler d'une autre manière est chez eux déshonorant. Mais ils sont justes ... » (F45 §43). 
vertus morales prêtées par Ctésias à des êtres proches de l'animal paraissent annoncer certains aspects du cynisme. C'est donc une conception de l'homme qui se joue dans ce tableau de l'Inde.

Univers spécifique et isolé par la forme monographique elle-même, l'Inde de Ctésias doit pourtant être resituée dans l'ensemble de son œuvre, puisque, s'il lui consacra un ouvrage, l'auteur composa une histoire vingt fois plus volumineuse des Empires successifs d'un Orient moins distant.

\section{LE VOLET D'UN DIPTYQUE ORIENTAL}

Au sein de l'œuvre de Ctésias, l'Inde s'inscrit dans un diptyque oriental, qui en fait à la fois l'opposé et le double de la Perse.

\section{L'Inde à l'opposé de la Perse}

Entre l'Inde et la Perse, le contraste est frappant. Elles s'opposent d'abord, dans l'esprit des Grecs, et dès Hérodote, comme deux objets ethnographiques distincts ${ }^{128}$ : alors que la Perse peut se voir et se visiter, l'Inde n'est connue que par ouï-dire ; alors que la Perse a une histoire, l'Inde n'est que nature et, par conséquent, la Perse est sujette aux changements quand l'Inde vit dans un éternel présent, celui des lois de la nature qui régissent la vie d'un peuple où nul individu ne se distingue ${ }^{129}$.

Mais, chez Ctésias, le contraste s'étend au plan moral et politique. Alors qu'en Perse les individus s'affrontent et s'entretuent, les Indiens vivent en harmonie. Sur le plan politique, le roi de Perse est constamment victime de trahisons et défections, tandis que les Indiens n'ont que dévouement pour leur roi ${ }^{130}$. Les mœurs royales ne s'opposent pas moins : en Inde, le roi n'a pas le droit de s'enivrer ${ }^{131}$; chez les Perses, au contraire, l'ébriété du roi peut assurer le succès d'une révolution de palais, puisque Xerxès II est assassiné pendant son sommeil "alors qu'il s'était enivré lors d'une fête " ${ }^{132}$. Sur le plan judiciaire, le roi des Indiens dispose d'une source dont l'eau fait dire la vérité, ce qui lui permet de condamner les coupables et d'acquitter les innocents ${ }^{133}$. En Perse règnent au contraire l'arbitraire et la condamnation des innocents, au point que,

128. Cf. D. LENFANT, art. cit. (n. 118).

129. Les rares individus mentionnés dans les fragments des Indica ne sont pas indiens : il s'agit d'un marchand bactrien ( $\mathrm{F} 45$ §6), du Roi de Perse et de sa mère ( $\mathrm{F} 45$ §9).

130. F45 $\$ 30$.

। 131. F50.

132. $\mathrm{F} 15 \S 48$.

133. F45 $\$ 31$. 
même lorsqu'il y a jugement, le verdict n'est pas nécessairement respecté par le Roi ${ }^{134}$. Quant à la mort, elle vient en Inde du climat ou de la faune, alors qu'en Perse ce sont les hommes qui la répandent.

Cela ne veut pas dire que l'Inde soit proposée en modèle, pas plus que l'épisode des Ethiopiens Longue Vie n'implique chez Hérodote un idéal monarchique. Mais le contraste revêt sans nul doute une valeur polémique. Pourtant, malgré cette opposition, ces deux mondes relèvent bien d'un même univers oriental.

\section{Les deux facettes d'un Orient cruel et aberrant}

L'Inde et la Perse se rejoignent dans l'image d'un Orient empreint de cruauté. Cette férocité est celle des hommes en Perse, celle des bêtes en Inde, mais la souffrance physique est également présente dans ces deux mondes et l'on pourrait établir un parallèle précis entre les supplices infligés en Perse et les souffrances dues à la faune ou aux poisons de l'Inde.

L'Inde et la Perse contribuent, chacune à leur manière, à faire de l'Orient le lieu de l'excès, l'antithèse du $\mu \eta \delta \dot{\varepsilon} v$ $\alpha$ $\gamma \alpha v$. L'extrême justice des Indiens est aussi démesurée que l'extrême injustice des Perses.

Enfin, l'Inde et la Perse sont toutes deux marquées au sceau de l'absurdité et de la confusion : en Inde, les espèces vivantes tendent à se confondre ; dans les Empires d'Orient, la confusion affecte les sexes : avec des femmes guerrières et puissantes comme Sémiramis ou Zarinaia ${ }^{135}$, avec des hommes efféminés tels que Sardanapale, Ninyas, Nanaros ou Parsondas, les hommes et les femmes de pouvoir échangent leurs attributs sexuels. Les repères habituels s'estompent finalement dès que l'on pénètre en Orient et l'écart ne fait qu'augmenter avec l'éloignement.

\section{CONCLUSION}

Les Indica se trouvent au confluent de plusieurs traditions littéraires grecques - qui, de l'épopée à Hérodote en passant par Scylax, se rapportaient à l'Inde ou aux confins du monde - et d'un milieu perse qui offrit au moins à l'auteur le spectacle de ses importations exotiques et de son iconographie fantastique, mais qui lui transmit sans doute aussi des récits à caractère

134. Ainsi, Gingè, acquittée par les juges, est condamnée à mort par le Roi (F27 §70).

135. On notera cependant que l'inversion n'est pas totale : les femmes viriles conservent les attributs qui les rendent fatales aux hommes. Ainsi, des nombreux amants de Sémiramis, éliminés après avoir servi, ou du suicide de Stryangée, repoussé par Zarinaia. 
légendaire. Néanmoins, la spécificité de l'ouvrage ne s'arrête pas à ses sources réelles, littéraires, picturales ou imaginaires. La personnalité de Ctésias, médecin séduit par les avatars physiques, pourvu d'une imagination féconde et évidemment désireux de surprendre, a joué un rôle déterminant dans la sélection, l'interprétation et l'assortiment de ces divers éléments : nul doute que les mêmes sources n'eussent pas conduit au même résultat dans l'œuvre d'Hérodote.

Il est évidemment abusif de parler d'ethnographie à propos de l'Inde de Ctésias, mais, si l'ouvrage est de peu d'intérêt pour l'indianiste, il n'en a pas moins sa place dans l'histoire culturelle de l'Occident. Il ne témoigne pas seulement de la manière dont les Grecs perçurent l'Extrême-Orient avant l'expédition d'Alexandre, car, au-delà de la conquête macédonienne, au-delà même de la disparition matérielle de l'ouvrage, la représentation qu'il propose ne fut pas sans de nombreuses survivances : les Indica laissèrent en héritage des schémas imaginaires qui, pour changer de sens, n'en perdurèrent pas moins. On a coutume d'évoquer leur riche descendance médiévale, en particulier les nombreux bestiaires, littéraires ou sculptés. Mais Ctésias est aussi, plus que tout autre, aux origines d'un Orient binaire, dont la structure marquait encore un Eugène Delacroix : la Mort de Sardanapale et la Femme indienne attaquée par un tigre n'incarnent-elles pas une fois de plus les deux facettes de cet Orient aussi cruel que fascinant?

Dominique LENFANT Université de Strasbourg 


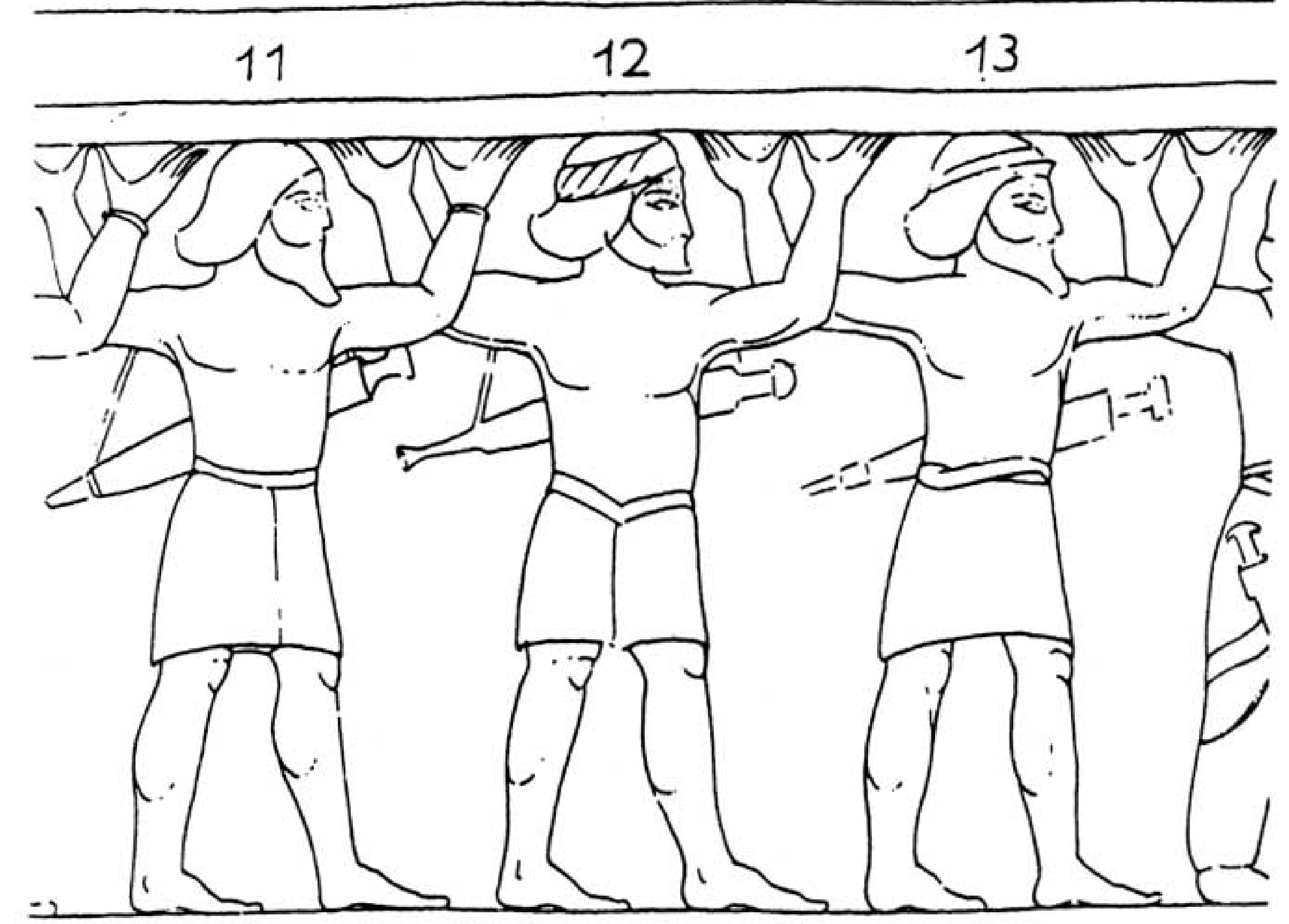

Fig. 1. Les Indiens comme porteurs de trône sur les tombeaux achéménides :

11. Sattagyde. 12. Gandarien. 13. Indien.

(G. WALSER, Die Volkerschaften..., Falttafel 1) 


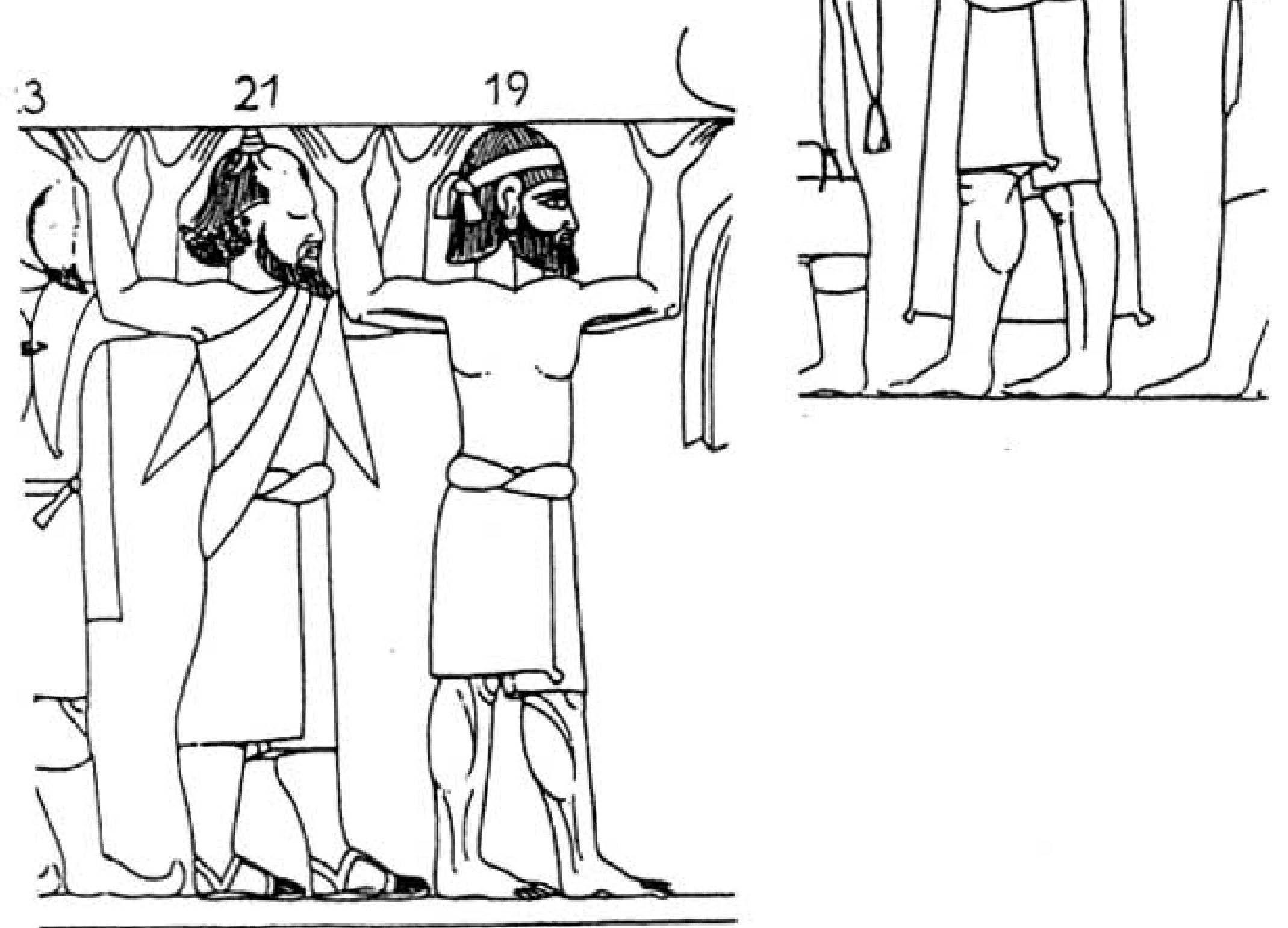

Fig. 2. Les Indiens comme porteurs de trône dans la salle aux cent colonnes de Persépolis : 21. Sattagyde ? 19. Indien? 14. Gandarien? (ibid., pp. 62-63) 


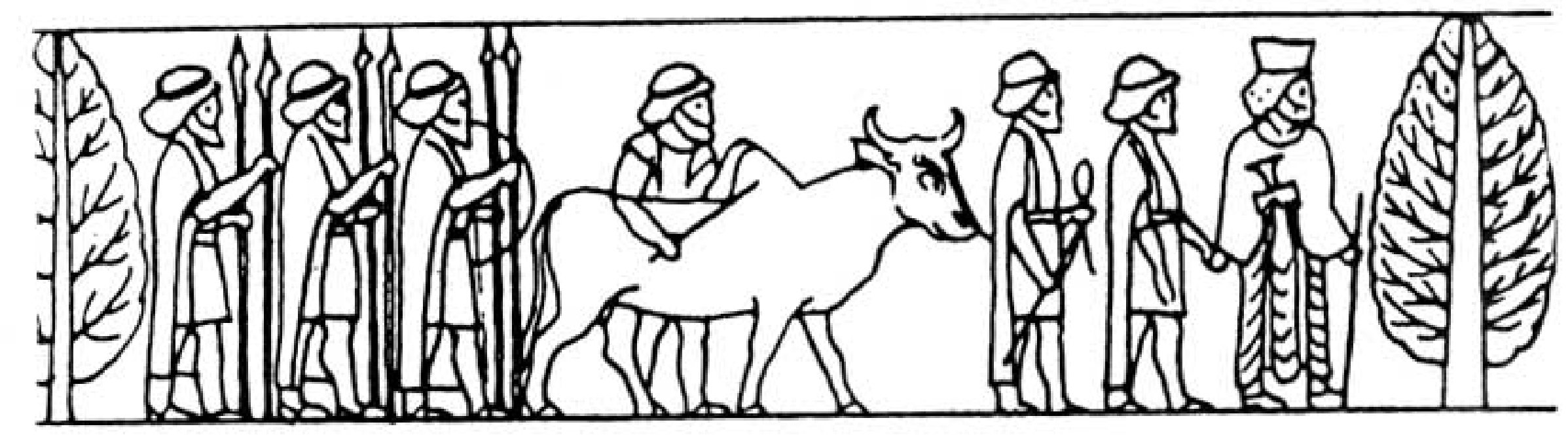

Fig. 3. Gandariens?

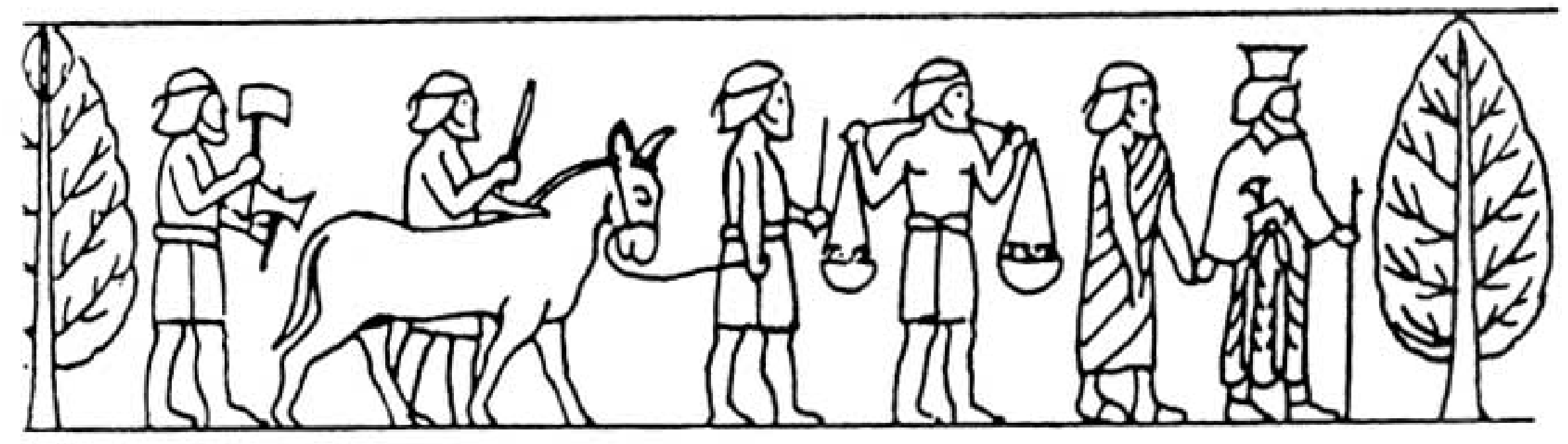

Fig. 4. Indiens?

Les Indiens comme porteurs de présents

à Persépolis

(ibid., Falttafel 2, délégations $\mathrm{n}^{\circ} 14$ et 18 ) 